\title{
Use OWC Control to Achieve Lamp Energy Consumption Comparison WANG De-yan
}

Wuxi Institute of Technology, Wuxi 214121, China

Wdyan297@sohu.com

\begin{abstract}
Keywords: Data Analysis, Statistic, OWC Drawing control, B/S Model, Chart Container
\end{abstract}
\begin{abstract}
When develop a B/S management system for the LED lamp company, there are many data need analysis on WEB Page. How to directly display lamp energy consumption, we use OWC control to draw the picture. The experimental results prove that the method is simple, effective.
\end{abstract}

\section{Introduction}

When we start to develop a management system for the LED lamp company, we do a lot of requirements analysis, and find use the picture shows the LED has lower energy is very directly. So we want to find a method that can get data from database, then display on WEB page. At present, in the majority of B/S structure information management software, users used the statistical chart to observation and analysis all kinds of data. This application often used in the form class program, if the graph function in WEB pages, there are some differences. Because if the WEB page use existing ActiveX statistical chart control, it will be direct embedded in webpage script, and require the client to download and install the appropriate control, so there will be more problem ,such as system installation and maintenance, distributed data access and so on. So let us consider a good pattern, we can let data operation and dynamic generation of statistical image through server, then release to the foreground. The client software configuration requirements are very low, as long as the foregrounds install the browser[1]. The author thinks that the OWC component is a good choice. OWC (Office Web Components) is a COM control set that released with Microsoft Office software. They not only can be used in WEB pages, VB forms controls in a container, but also can be used as an invisible objects in memory. The author will integrate several techniques on how to use OWC components development WEB statistical control charts. Use the LED lamp's energy consumption comparison diagram as the carrier, to achieve chart and carry on data analysis.

\section{Design philosophy}

The basic idea is running the OWC Chart Components on the server, using its abundant programming interface to the data source, and set up chart's format and type, use the Export Picture method to derive a temporary GIF files, final combined with the ASP.NET technology, return the GIF image to the client browser.

Use a two-dimensional chart to descript, $\mathrm{X}$ axis descript power and $\mathrm{Y}$ axis descript energy, the data refer the Table 1:

Table 1.

THE ORIGINAL DATA

\begin{tabular}{|l|l|l|}
\hline POWER & ENERGY & TYPE \\
\hline 1 & 85 & 1 \\
\hline 3 & 245 & 1 \\
\hline 5 & 450 & 1 \\
\hline 7 & 650 & 1 \\
\hline
\end{tabular}




\begin{tabular}{|l|l|l|}
\hline 10 & 950 & 1 \\
\hline 15 & 1450 & 1 \\
\hline 18 & 1750 & 1 \\
\hline 21 & 1900 & 1 \\
\hline 25 & 2300 & 1 \\
\hline 10 & 65 & 2 \\
\hline 15 & 101 & 2 \\
\hline 25 & 198 & 2 \\
\hline 40 & 340 & 2 \\
\hline 60 & 540 & 2 \\
\hline 100 & 1150 & 2 \\
\hline 150 & 1840 & 2 \\
\hline 200 & 2660 & 2 \\
\hline 300 & 4350 & 2 \\
\hline 500 & 7700 & 2 \\
\hline
\end{tabular}

Note: TYPE 1 represent LED lamp, TYPE 2 represent incandescent lamp.

The author found that the real difficulty lies not in how to use the OWC interface, but how to send the graphics to the client browser? Now we will step by step to building a custom WebChart control.

\section{References in the program owc}

A. Add reference

Build a website called WebOwc. Add OWC reference: in the root directory of the web site "Add Reference", select "Microsoft Office 11.0 Object Library" or directly select the path for the C:\Program Files\Common Files\Microsoft Shared \Web Components $\backslash 11$ lowc11 owc11 in the.Com tab;

B. Inheritance of the WebOwc base class, add a using reference

Asp.net arranged all the built-in objects under a different directory, the directory is the namespace, and when to use the.Net built-in objects, we must refer to the object namespace. The difference between using and add reference is add reference just for add assembly, one or a few assembly is added to the reference of a project to a solution. Then, you can use these references in the classes in the project, through the use of using + space + has been cited in the assembly of a class corresponds to the namespace.

For example:

Using Microsoft.Office .Interop.Owc11;

\section{Examples of OWC contrails}

How to analysis of dynamic data is the key of use the OWC control. So it must be with the database as the carrier, data source refer Table 1

Note:use LED lamp's energy consumption data as example, set TYPE as ' 1 '.

A. Database connection

...database connection:

string string1 = "SELECT Gongl,Nengh FROM Led where TYPE="' + 1+ "'";

SqlConnection database connectionobject 
= new SqlConnection ( database connectionstring);

Database connectionobject. Open(); //open database

SqlDataAdapter data adapter = new SqlDataAdapter(string, database connectionobject);

DataSet dataset $=$ new DataSet();

Data adapter.Fill(dataset);

B. For the $\mathrm{X}$ and $\mathrm{Y}$ axes parameters

Draw a diagram to $\mathrm{X}$ axis and $\mathrm{Y}$ axis.OWC diagram data values cannot be directly removed from the dataset, so first remove data values from the dataset, and then put the data into two one-dimensional arrays, respectively as the $\mathrm{X}$ and $\mathrm{Y}$ axis value. The number of array elements can be determined according to the number of records in the actual.

Save data:

string [] X arrayname1 = new string[9];

string [] Y arrayname2 = new string[9];

Evaluate for two array:

For (int $\mathrm{i}=0$; $\mathrm{i}<$ data set.Tables[0].Rows.Count; $\mathrm{i}++$ )

\{

Xarrayname1 [i] =data set.Tables[0].Rows[i][0]（which one feild）.ToString();

\}

Yarrayname2 [i] =data set.Tables[0].Rows[i][1]（which one feild ) .ToString();

Specific string for $\mathrm{X}$ axis in order to display data appoint:

String Xstring1 = String.Empty;

Foreach (string strData in X arrayname1)

\{

\}

Xstring1+= strData + "\t";

Specific string for $\mathrm{Y}$ axis in order to corresponding to the $\mathrm{X}$ axis:

String Ystring2= String.Empty;

Foreach (string strValue in Y arrayname2)

\{

\}

Ystring2 += strValue + "\t";

C. The OWC control drawing

First create a Chart Space object to place the chart

ChartSpace Chartobject $=$ new ChartSpaceClass();

Add the chart object in Chart object

ChChart InsertChart = Chartobject. Charts.Add(0);

Then according need to specify the type of chart, such as line graphs and pie charts and histograms and so on.

InsertChart.Type = ChartChartTypeEnum.chChartTypeLine; //line graph

InsertChart.HasLegend = false;

If you need to add the title for chart, please set HasTitle as "true"

InsertChart.HasTitle = true;

If you want add a title for the chart, you should do the next step:

InsertChart.Title.Caption = "LED ";

Add the illustration for the $\mathrm{X}$ and $\mathrm{Y}$ axes:

InsertChart.Axes[0].HasTitle = true; 
Xaxis InsertChart.Axes[0].Title.Caption = "POWER";

InsertChart.Axes[1].HasTitle = true;

InsertChart.Axes[1].Scaling.SplitMinimum = 200;

Yaxis InsertChart.Axes[1].Title.Caption = "ENERGY";

According to the needs, set one or more chart in a container, if only one please set"InsertChart.SeriesCollection.Add(0)",if need two please set "InsertChart.SeriesCollection.Add(1)",and so on,for example only one:

InsertChart.SeriesCollection.Add(0)

InsertChart.SeriesCollection[0].SetData(ChartDimensionsEnum.chDimSeriesNames, +(int)ChartSpecialDataSourcesEnum.chDataLiteral, strSeriesName);

Set categories(Xaxis):

InsertChart.SeriesCollection[0].SetData(ChartDimensionsEnum.chDimCategories, +(int)ChartSpecialDataSourcesEnum.chDataLiteral, Xstring1);

Set data(Yaxis):

InsertChart.SeriesCollection[0].SetData(ChartDimensionsEnum.chDimValues, (int)ChartSpecialDataSourcesEnum.chDataLiteral, Ystring2);

D. output .gif picture

The OWC chart based on the "drawing room". So when draw the icons, first create a chart space, on the space can draw one or more chart. Drawing space can use a variety of forms such as Chart holder and placeholder,you can also can directly output the picture to Image. This paper use image to display picture.

Output file: string outpath=( Server.MapPath(".")) + "\|picturefile ";

laySpace.ExportPicture(outpath, "picturetype, GIF", 309, 250);

create .gif file path string strRelativePath = "./picturefile";

Display picturefile:

String strImageTag = "<IMG SRC="' +outpath + "'/>";

PlaceHolder1.Controls.Add(new LiteralControl(strImageTag));

close database.

\section{Draw a picture}

Chart result:

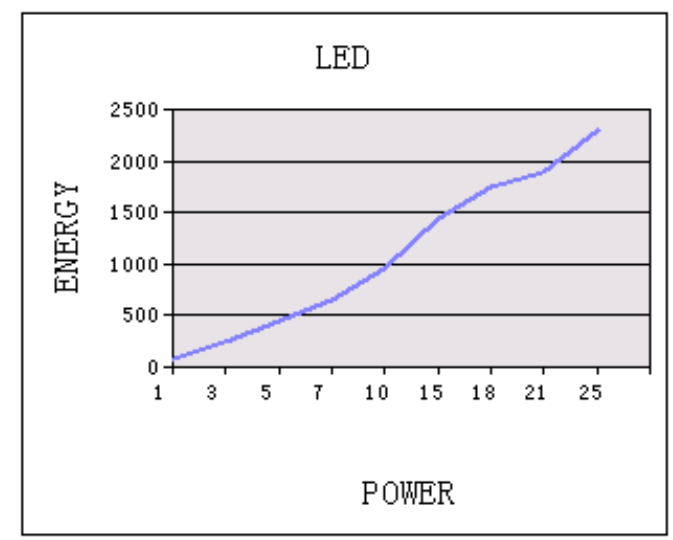

Fig. 1 LED lamp energy diagram

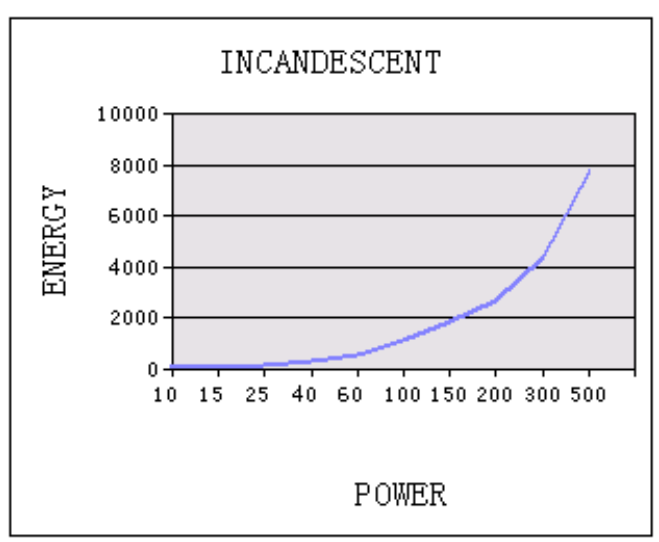

Fig. 2 Incandescent energy diagram 
If you want to add more chart in chart container ,you can design many Xaxis and Yaxis data,then add series, InsertChart.SeriesCollection.Add(1);also you need add "Set categories(Xaxis)" and "Set data(Yaxis)”,InsertChart.SeriesCollection[1] ～，～InsertChart.SeriesCollection[1] ～， InsertChart.SeriesCollection[1].

If you want set chart type, you can get it from OWC.ChartChartTypeEnum, often we shoud select from chChartTypeLine , chChartTypeArea , chChartTypeBarClustered , chChartTypeColumnClustered and so on.

As we see, the picture directly displays the energy of LED lamp. In the paper, the method of using OWC control is very simple.

\section{References}

[1] Yu Yun,Wei Jin,Yang Bo.Design and Implementation of Flexible Dynamic Report Generator Based on OWC[J].Computer Applications and Software2009(12)93-95

[2] XIAO Zhong-jie.OWC Designs Medium Application Study In The Dynamic State Website[J].Journal of Qinghai Normal University(Natural Science Edition).2010(01)70-72

[3] DING Jian-bo,YANG Kun.Graphical Development and Application of OWC series[J]. Computer Era. 2011(01)33-35

[4] S.Patel, K.Ram,M.Rafiuddin Ahmed. Effect of Partial Blockage of Air Duct Outlet on Performance of OWC Device[J].Journal of Central South University. 2012(02)748-753

[5] LIN Yong-xing,XU Xiao-yan. A Dynamic Creating Chart Method Based on OWC in Web Page [J] .Computer and Modernization.2010 (01)153-155

[6] LI Xing-du.Data Collection and Statistics System of Network Learning Behavior [J].Journal of Lanzhou Polytechnic College,2012 (05) 